\title{
Data analysis preparation and characterization of porous manganese oxide films for super capacitor by cathodic electrode position
}

\author{
Kun Liang, Mei Zhou, Wencheng Hu \\ State Key Laboratory of Electronic Thin Films and Integrated Devices, University of Electronic \\ Science \& Technology of China, Chengdu 610054, P. R. China
}

Keywords: Porous, Manganese oxide film, Supercapacitor.

\begin{abstract}
Porous manganese oxide film for electrochemical supercapacitors is electrodeposited directly onto a nickel sheet by cathodic electrodeposition in a KMnO4 aqueous solution. The obtained film is characterized by X-ray diffraction (XRD), energy dispersive spectroscopy (EDS), scanning electron microscopy (SEM), cyclic voltammetry $(\mathrm{CV})$ and galvanostatic charge/discharge test. The SEM images showed the formation of porous structure on the surface of nickel substrate. The CV curves are tested in a Na2SO4 aqueous solution, showing double layer capacitive behavior in the potential window of $-0.2 \sim 1.0 \mathrm{~V}$ versus SCE. The highest specific capacitance is $165.7 \mathrm{~F}$ g-1 with a scan rate of $1 \mathrm{mV} \mathrm{s}-1$.
\end{abstract}

\section{Introduction}

Supercapacitor is a new energy storing device [1-2] between chemical power source and electrostatic condenser. Supercapacitor has the advantage of high power density, rapid charging/discharging, longer service time and good temperature characteristics. Therefore, it is a new kind of efficient and practical energy storage device.

Currently, much attention is paid on power density, energy density and cycling performance, which are the key parameters for energy storage devices, since the demand for power sources delivering energy in the high-power or pulse-power form has increased [3]. The previous study of electrochemical capacitor is mostly involved in the two aspects: electrode materials and electrolyte solutions, which are the main factors determining the performances of electrochemical capacitor.

Many materials have been used as electrode materials. They can be divided into three categories: carbon materials [4-8], conductive polymers [9-10] and transition metal compounds [11-14]. As electrochemical capacitor electrode materials, metal oxides produce electrode potential to charge the capacitance, mainly by highly reversible chemical suck and take off redox reaction [15].

Manganese is a kind of transition metals due to its ion exchange, molecular adsorption, catalytic, electrochemical and magnetic properties [16-17]. Manganese element can be presented in three different valence states and its oxides are highly complex. Manganese dioxide is a material with the advantage of high specific capacitance, wide use as catalysts and molecular-sieves, and low cost of raw materials. Therefore, the research of manganese oxide has been the hot point.

In the present paper, the manganese oxide films for electrochemical supercapacitors were obtained by cathodic electrodeposition from a $\mathrm{KMnO} 4$ aqueous solution, controlling the deposition time and the current density. The as-prepared films were characterized by energy dispersive spectroscopy (EDS), scanning electron microscopy (SEM) and X-ray diffraction (XRD). Moreover, the electrochemical performances were also detected by cyclic voltammetry $(\mathrm{CV})$ and galvanostatic charge/ discharge test.

\section{Experiment}

\section{Preparation of $\mathrm{MnO2}$ film}

Prior to the deposition, nickel sheet used as the substrate was polished in an acidic solution, containing concentrated HNO3, H3PO4 and glacial acetic acid with a volume ratio of 2:6:3. The electrolyte of electrodeposition was prepared with analytical pure $\mathrm{KMnO} 4$ and was adjusted the $\mathrm{pH}$ value to 1 by adding concentrated $\mathrm{H} 2 \mathrm{SO} 4$. 
The $\mathrm{MnO} 2$ films were electroplated directly onto $1 \times 3 \mathrm{~cm} 2$ nickel sheet by cathodic electrodeposition from the $0.2 \mathrm{M} \mathrm{KMnO} 4$ aqueous solution at a current density of $0.2 \mathrm{~A} \mathrm{dm}-2$ and $90^{\circ} \mathrm{C}$ by the galvanostatic method. Moreover, the anode was a flat-plate carbon, and the deposition time was 5 minutes. The as-deposited films were thoroughly washed with deionized water.

\section{Apparatus}

A PS-3005D power supply (Zhaoxin Electronic Equipment \& Instrument Corporation Shenzhen, China) was used to electrodeposit $\mathrm{MnO} 2$ films. The specific capacitance of the sample was detected by cyclic voltammetry and galvanostatic charge/discharge test in an electrochemical workstation (CHI-660D, Chenhua Instrument, China). During the measurement of electrochemical performances, a standard three-electrode system was employed, including a platinum wire (1 mm diam), a $\mathrm{Hg} / \mathrm{Hg} 2 \mathrm{Cl} 2$ electrode ( $\mathrm{SCE}$, saturated $\mathrm{KCl}, 0.214 \mathrm{~V}$ vs $\mathrm{NHE}$ at $25{ }^{\circ} \mathrm{C}$ ), a prepared $\mathrm{MnO} 2$ film as counter electrode, reference electrode and working electrode, respectively. All the electrodes were immersed in electrolyte. And the measurement process was finished in a $1 \mathrm{M} \mathrm{Na} 2 \mathrm{SO} 4$ aqueous solution, at room temperature and normal pressure. The data were collected by the electrochemical workstation.

\section{Results and discussion}

Fig. 1(a) shows the XRD patterns of the sample, in which only diffraction peaks of $\mathrm{MnO} 2$ and substrate can be observed, indicating that only the $\mathrm{MnO} 2$ film is formed on nickel substrate. The distinctive diffraction peaks for the sample is located at $37.9^{\circ}, 65.1^{\circ}$ and $77.8^{\circ}$, respectively. These peaks can be indexed to ramsdellite $\mathrm{MnO} 2$ (PDF\#42 - 1316), and the corresponding crystalline faces are (111), (020) and (313). Moreover, the lattice contents are $a=9.531 \AA, b=2.864 \AA, c=4.7 \AA$ and $\alpha=\beta=\gamma=90^{\circ}$. According to the Scherrer formula, the grain size is calculated to be $24.3 \mathrm{~nm}$.
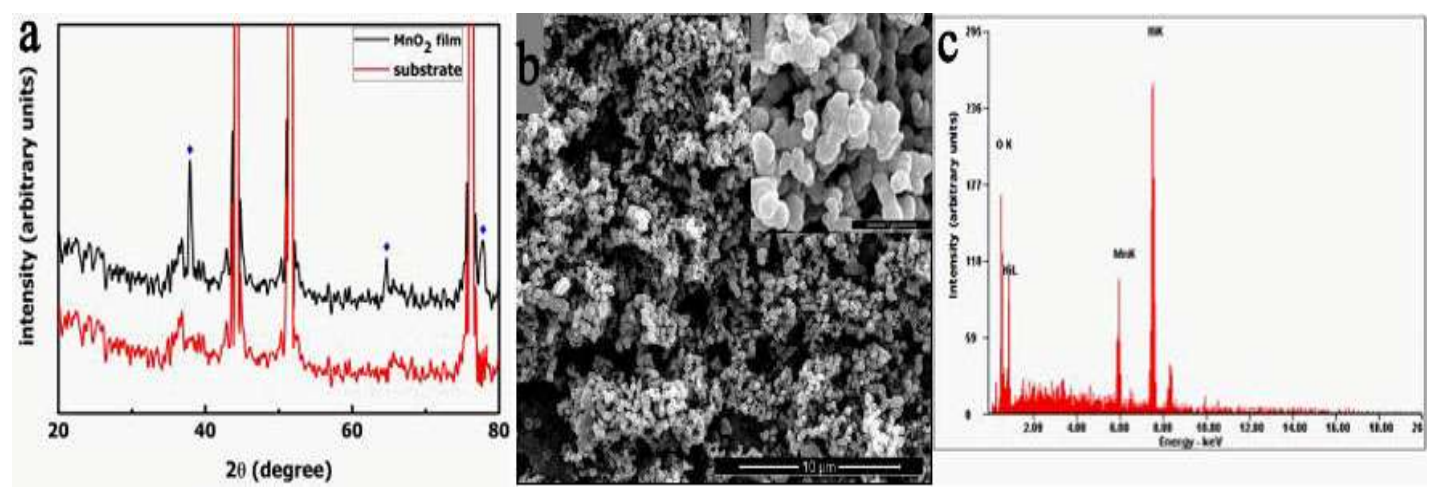

Fig. 1: (a) XRD pattern of the as-prepared MnO2 film; (b) SEM images with different magnification of the MnO2 film; (c) EDS spectrum of the $\mathrm{MnO} 2$ film.

The morphologies of the $\mathrm{MnO} 2$ films can be observed in Fig. 1(b). The clusters appear as interleaving sheets or flakes. These clusters arrange in order to form porous structure. The reason for the formation of the porous structure may be as follow. On the cathode, the current efficiency is very low in the conversion of the metal ions valence from high to low. Because the electrolyte is acidic, hydrogen ions in the solution are reducted to hydrogen atoms and hydrogen, which are adsorbed on the substrate surface to prevent the deposition of $\mathrm{MnO} 2$. However, the surface of the substrate does not been completely occupied by hydrogen atoms and hydrogen. There is still $\mathrm{MnO} 2$ deposit growing heterogeneously to form the porous structure as shown. The size of the deposit is about $217 \mathrm{~nm}$, consisting of several crystal grains.

To analyse the detail component of the sample, the EDS measurement is carried out. The EDS data show that the film contains $\mathrm{O}, \mathrm{Mn}$ and $\mathrm{Ni}$ elements in Fig. 1(c). The image gives further evidence to the composition of the product. The surface of the film consists of small nanoparticles and the deposit is a very thin layer on the substrate. Therefore, the intension of Ni is higher than $\mathrm{Mn}$ and $\mathrm{O}$. 

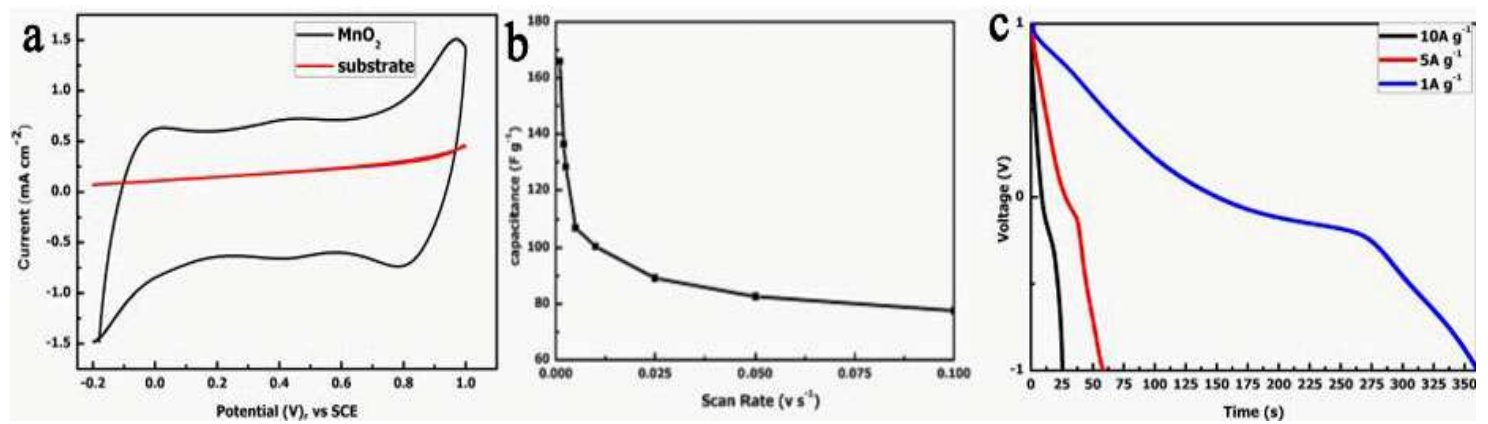

Fig. 2: (a) The cyclic voltammograms of the obtained film and nickel substrate; (b) Capacitance vs scan rate for a sample; (c) Typical discharge curves for the as-prepared $\mathrm{MnO} 2$ film in the potential range of $-1.0 \sim 1.0 \mathrm{~V}$.

Fig. 2(a) explains that the cyclic voltammogram of the $\mathrm{MnO} 2$ film tested in the $1 \mathrm{M} \mathrm{Na} 2 \mathrm{SO}$. The image shows double layer capacitive behavior within the potential range of $-0.2 \sim 1.0 \mathrm{~V}$ versus $\mathrm{SCE}$. The highest specific capacitance is $165.7 \mathrm{~F}$ g- 1 with a scan rate of $1 \mathrm{mV} \mathrm{s}-1$. In Fig. 2(a), the voltammogram of the substrate in red line is also tested in the same system as the obtained films. The voltammogram exhibits the ideal capacitive characteristics and displays voltammetric current remains nearly constant.

Fig. 2(b) displays the specific capacitance values with different scan rates and summarizes the effect of scan rate on specific capacitance. The image shows a decrease of specific capacitance with increasing scan rate following a logarithmic correlation. The specific capacitance is $77.56,82.62$, $89.18,100.47,107.08,128.27,136.36$ and $165.85 \mathrm{~F} \mathrm{~g}-1$ with the potential scan rate of $100,50,25$, $10,5,2.5,2$ and $1 \mathrm{mV} \mathrm{s}-1$, respectively.

Galvanostatic charge/discharge experiment is performed at current densities of 10, 5 and $1 \mathrm{~A} \mathrm{g-1.}$ The discharge curves of $\mathrm{MnO} 2$ film are shown in Fig. 6, indicating a good capacitive behavior. Fig. 2(c) exhibits the discharge time at different current densities to calculate specific capacitance values. The specific capacitance value is $181 \mathrm{~F} \mathrm{~g}-1$ with the scan rate of $1 \mathrm{~A}$ g- 1 . The specific capacitance value is larger than that obtained in the cyclic voltammetry. The reason may be that the ions and electrons have enough time to deliver on the interface and surface of the film during the faradic redox reaction.

\section{Conclusion}

Porous manganese oxide films have been obtained by cathodic electrosynthesis from the KMnO4 aqueous solution. The results of XRD, EDS, CV, SEM and galvanostatic charge/discharge test indicated the formation of porous manganese oxide films. SEM showed the porosity of as-prepared film. The XRD patterns of the samples demonstrated that the film is the ramsdellite $\mathrm{MnO} 2$ with a grain size of $24.3 \mathrm{~nm}$. Cyclic voltammetry of the obtained film shows that the high specific capacitance (SC) is $165.7 \mathrm{~F} \mathrm{g-1}$ with a scan rate of $1 \mathrm{mV} \mathrm{s}-1$, while the result of galvanostatic charge/discharge test is $181 \mathrm{~F} \mathrm{~g}-1$. And the double layer capacitive behavior is in the larger voltage window of $-0.2 \sim 1.0 \mathrm{~V}$ versus SCE. The obtained samples showed the high power-energy characteristics and have a potential application in electrode materials of the supercapacitor.

\section{References}

[1] Prasad, K.R, Miura, N. Electrochemical synthesis and characterization of nanostructured tin oxide for electrochemical redox supercapacitors. Electrochem. Commun. 6(8), pp. 849-852, 2004.

[2] Lee, K.T. Wu, N.L. Manganese oxide electrochemical capacitor with potassium poly(acrylate) hydrogel electrolyte. J. Power Sources. 179(1), pp. 430-434, 2008.

[3] Hu, C.C. Wang, C.C. Nanostructures and Capacitive Characteristics of Hydrous Manganese Oxide Prepared by Electrochemical Deposition. J. Electrochem. Soc. 150(8), pp.1079-1084, 2003. 
[4] Pandolfo, A.G. Hollenkamp, A.F. Carbon properties and their role in supercapacitors. J. Power Sources. 157(1), pp.11-27, 2006.

[5] Gambya, J. Tabernaa, P.L. Simona, P. Fauvarquea, J.F. Chesneaub, M. Studies and characterisations of various activated carbons used for carbon/carbon supercapacitors. J. Power Sources. 101(1), pp.109-116, 2001.

[6] Min, M. Machida, K. Jang, J.H. Naoi, K. Hydrous RuO2/Carbon Black Nanocomposites with 3D Porous Structure by Novel Incipient Wetness Method for Supercapacitors. J. Electrochem. Soc. 153(2), pp.334-338, 2006.

[7] Kim, C. Choi, Y.O. Lee, W.J. Yang, K.S. Supercapacitor performances of activated carbon fiber webs prepared by electrospinning of PMDA-ODA poly(amic acid) solutions. Electrochimica. Acta. 50(2-3), pp.883-887, 2004.

[8] Saliger, R. Fischer, U. Herta, C. Fricke, J. High surface area carbon aerogels for supercapacitors. J. Non-Cryst. Solids. 225, pp. 81-85, 1998.

[9] Sharma, R.K. Rastogi, A.C. Desu, S.B. Manganese oxide embedded polypyrrole nanocomposites for electrochemical supercapacitor. Electrochimica. Acta. 53(26), pp.7690-7695, 2008.

[10] Laforgue, A. Simon, P. Sarrazin, C. Fauvarque, J.F. Polythiophene-based supercapacitors. J. Power Source. 80(1-2), pp.142-148, 1999.

[11] Pang, S.C. Anderson, M.A. Chapman, T.W. Novel Electrode Materials for Thin - Film Ultracapacitors: Comparison of Electrochemical Properties of Sol - Gel - Derived and Electrodeposited Manganese Dioxide. J. Electrochem. Soc. 147(2), pp.444-450, 2000.

[12] Hu, C.C. Tsou, T.W. Ideal capacitive behavior of hydrous manganese oxide prepared by anodic deposition. Electrocchem. Communn. 4(2), pp.105-109, 2002.

[13] Nam, K.W. Yoon, W.S. Kim, K.B. X-ray absorption spectroscopy studies of nickel oxide thin film electrodes for supercapacitors. Electrochimica. Acta. 47(19), pp.3201-3209, 2002.

[14] Grupioni, A.A.F. Arashiro, E. Lassali, T.A.F. Voltammetric characterization of an iridium oxide-based system: the pseudocapacitive nature of the $\mathrm{Ir} 0.3 \mathrm{Mn} 0.7 \mathrm{O} 2$ electrode. Electrochimica. Acta. 48(4), pp.407-418, 2002.

[15] Toupin, M. Brousse, T. Belanger, D. Influence of Microstucture on the Charge Storage Properties of Chemically Synthesized Manganese Dioxide. Chem. Mater. 14(9), pp.3946-3952, 2002.

[16] Ma, S.B. Ahn, K.Y. Lee, E.S. Oh, K.H. Kim, K.B. Synthesis and characterization of manganese dioxide spontaneously coated on carbon nanotubes. Carbon. 45(2), pp.375-382, 2007.

[17] Adelkhani, H. Ghaemi, M. Jafari, S.M. Novel Nanostructured MnO2 Prepared by Pulse Electrodeposition: Characterization and Electrokinetics. J. Mater. Sci. Technol. 24(6), pp.857-862, 2008. 